\title{
Determination of the fatigue behavior of coatings by means of an improved impact testing evaluation procedure
}

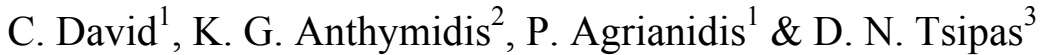 \\ ${ }^{I}$ Mechanical Engineering Department, Technical University of Serres, \\ Greece \\ ${ }^{2}$ Materials Department in Applied Research Center of Serres, Greece \\ ${ }^{3}$ Mechanical Engineering Department, \\ Aristotle University of Thessaloniki, Greece
}

\begin{abstract}
Impact testing is an efficient experimental procedure that enables the determination of the fatigue resistance of mono- and multilayer coatings deposited on various substrates, which is not possible with the common testing methods previously available. In this paper an advanced impact tester, capable of assessing the fatigue failure resistance of coatings working under cyclic loading conditions, is presented. The fatigue failure of the tested coating was determined by means of scanning electron and optical microscopy. The test results were recorded in diagrams containing the impact load versus the number of successive impacts that the tested coating can withstand.

Keywords: thin films and coatings, materials characterization, fatigue.
\end{abstract}

\section{Introduction}

The impact test method has been introduced as a convenient experimental technique to evaluate the fatigue strength of coatings being exposed in alternate impact loads [1-4]. According to this method a coated specimen is cyclically loaded by a hard ball that repetitively impacts on the specimen surface. The superficially developed Hertzian pressure induces a complex stress filled within the coating, as well as, in the interfacial zone. Both these stress states are responsible for distinct failure modes, such as a cohesive or adhesive one. The 
exposure of the layered compounds against impulsive stresses creates the real conditions for the appearance of coating fatigue phenomena based upon structural transformation, cracking generation and cracking growth, which are responsible for the gradual microchipping and the degradation of the coating.

\section{Experimental procedure:}

In this research coatings were characterized using an advanced impact tester system, which is shown in Fig.1. The system consists of three main parts:

- The main test device (centre)

- The power supply unit (left)

- The evaluation and controlling unit (right)

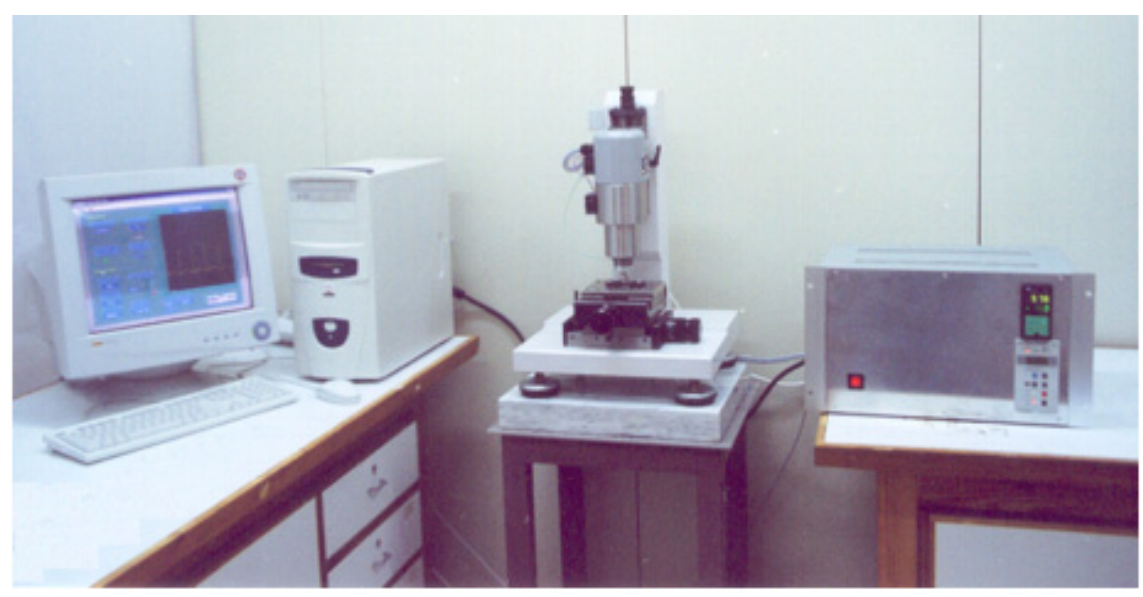

Figure 1: Impact tester system.

In the present paper characterization of coatings were carried out in such a system. This experimental set up is simple and user friendly and allows the determination of the fatigue behavior of a wide range of single and multielement coatings. The working principle of the impact tester is presented in figure 2 and is based on the alternate Laplace magnetic forces produced by the electromagnetic field, which is induced within the mechanical unit.

In order to make the impact tester system more efficient we redesigned the mechanical unit using finite elements to achieve the optimum magnetic flux density, which gives the higher magnetic force of the electromagnetic field and correspondingly an increased impact load (figure 3). Further more the control and the monitoring of the impact tester was improved. All four most important test parameters, the induced impact force, number of successive impacts, the impact frequency and the level of the coil temperature are monitored throughout 
each experiment. The whole mechanical apparatus is flexible and allows the operator to modify the desirable total number of impacts and impact force during the test procedure easily via the front panel of the evaluation and controlling unit (figure 4).

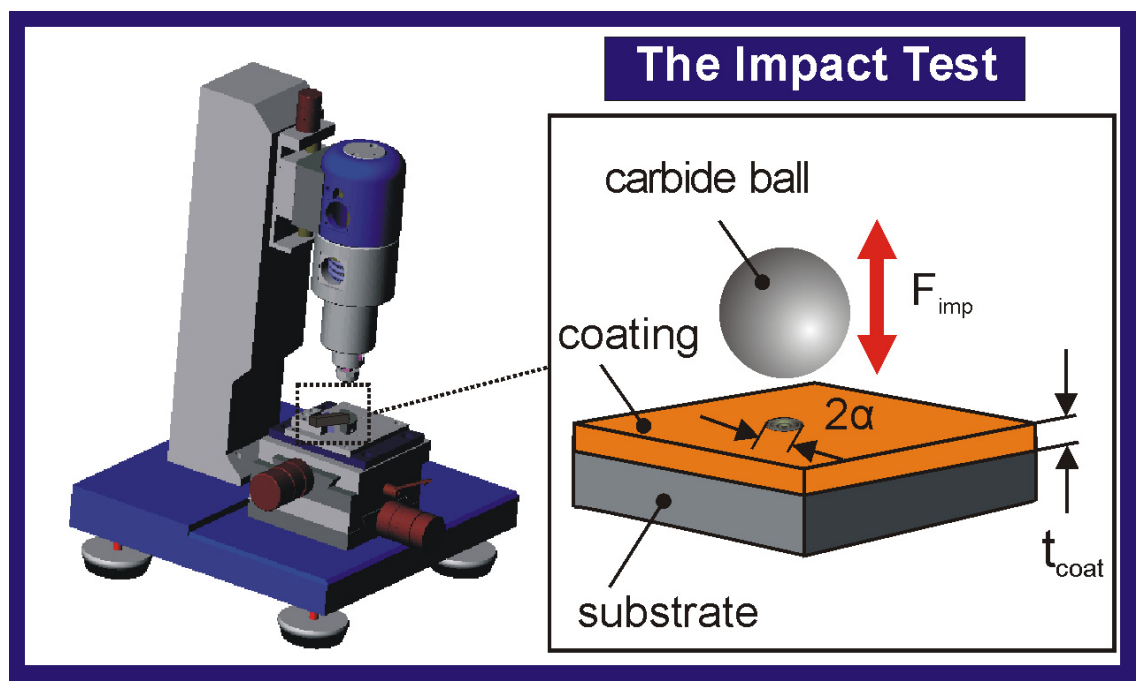

Figure 2: $\quad$ Impact tester working principles.

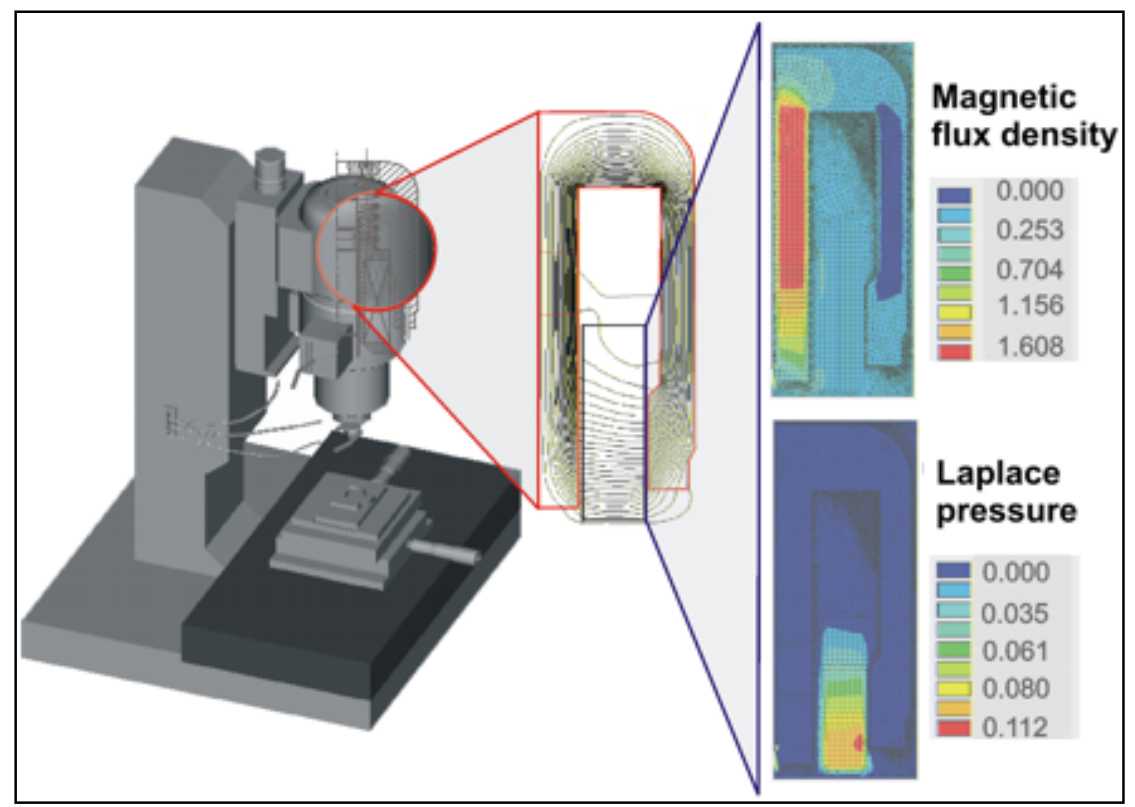

Figure 3: Increased impact load due to design optimisation. 
16 Computational Methods and Experiments in Materials Characterisation III

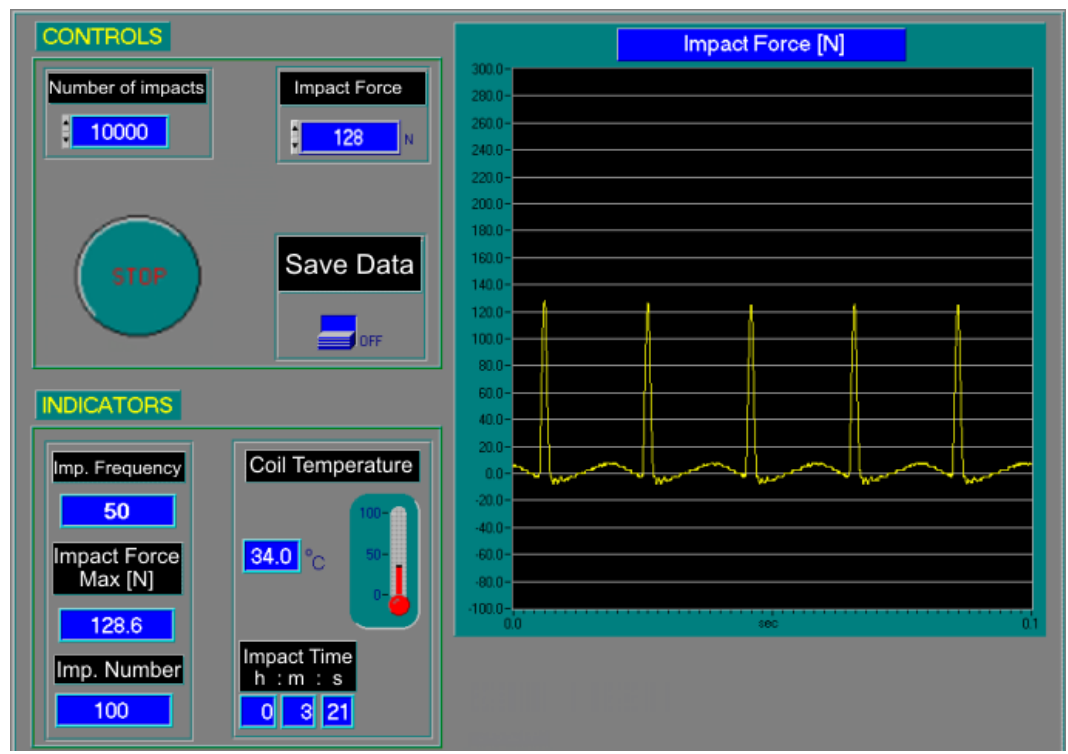

Figure 4: Front panel of the evaluation and controlling unit.

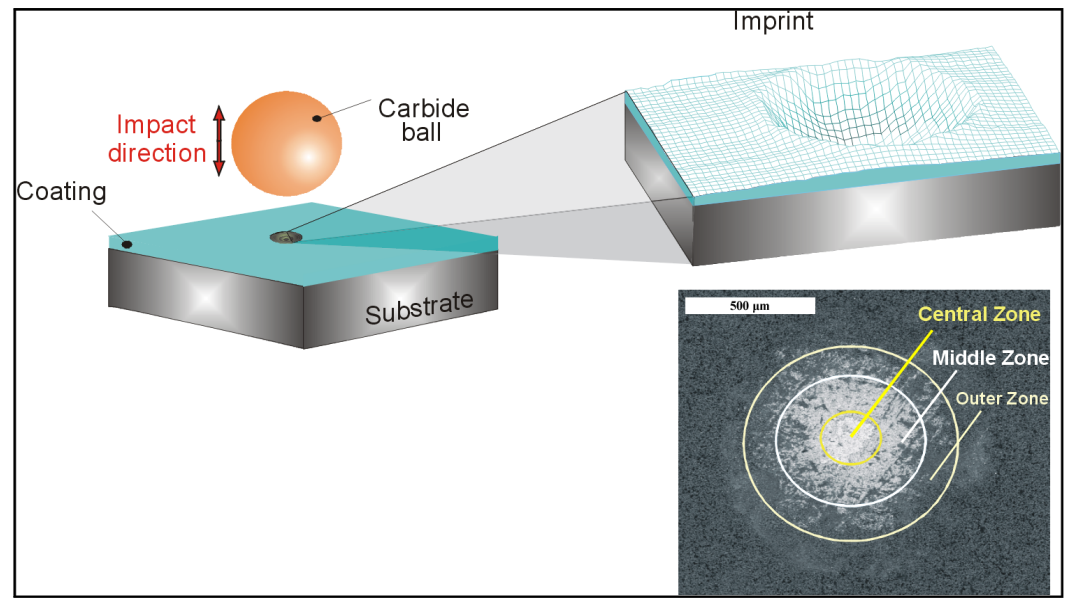

Figure 5: Impact crater with the developed coating failure.

The stress strain problem related to the impact test is the Herzian contact, which develops between the spherical indentor (carbide ball) and the examined layered space. Gradual intrinsic coherence release and coating microchipping or abrupt coating fracture and consequent exposure of the substrate material designate the coating failure. In all impact craters resulted from the experiments three different zones inside the impact cavity were identified (figure 5). A central zone in the mid of the impact cavity, where the coating is strained with 
compressive stresses and a gradual cohesive degradation takes place. The intermediate zone inside the piled up rim formed around the impact cavity, where tensile and shear stresses are building up and both cohesive and adhesive delamination arises. Finally, the peripheral zone of the impact cavity, where macrocracks might propagate and coating failure occurs.

The coating failure mode and its extent were assessed by SEM observations and EDX analysis. The contact load leading to coating fatigue fracture was recorded in diagrams (endurance strength curves) versus the number of impacts (figure 6). The impact load for which the coating after $10^{6}$ impacts do not fail is called limit of continues endurance of the coating.

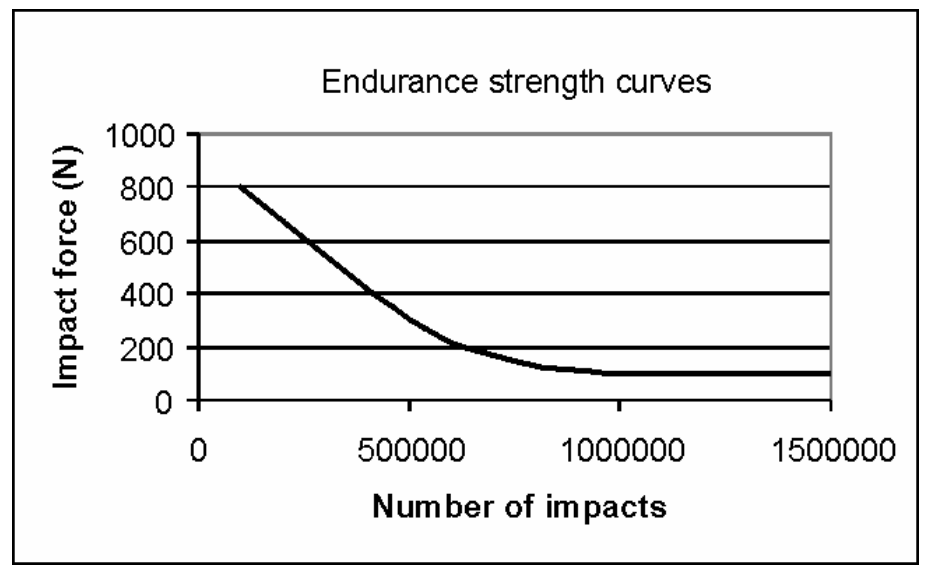

Figure 6: Typical endurance strength curve.

\section{Results and discussion}

In figure 7 the high cycle fatigue diagram of a Al, Fe pack coating on P92 steel ( $9 \%$ w.t. Cr, $1.8 \%$ w.t. W) substrate is shown. This coating consists of an outer Fe14A184 layer and an inner FeA113 layer. From impact testing procedure it was concluded that its limit of continues endurance was $100 \mathrm{~N}$ (Fig.8, 9). The main failure of the examined coating-substrate compound occurred in the central zone of the impact crater with coating degradation.

\section{Conclusions}

The work presented here shows a step forward in understanding the failure mechanisms of pack coatings. More specifically the paper reports the results of a novel experimental approach adapted to investigate the endurance performance of coating systems with refer to their mechanical properties and to deliver a semi-empirical design approach. Current impact testing investigations revealed the fatigue strength of Al, Fe pack coating on P92 steel substrate. 
18 Computational Methods and Experiments in Materials Characterisation III

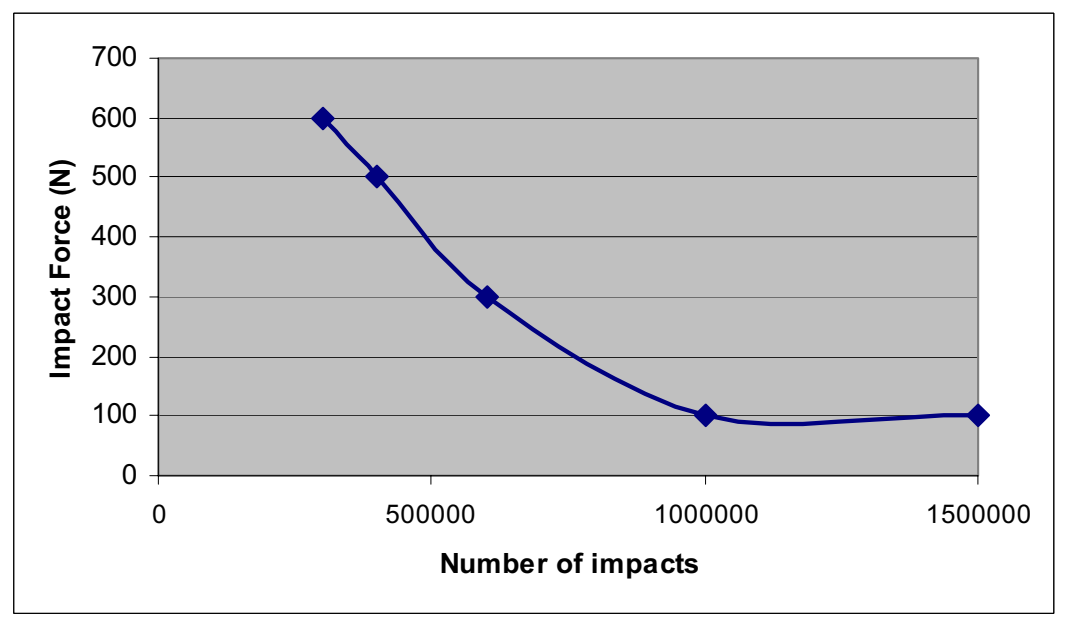

Figure 7: Endurance strength curve of Al, Fe pack coating on P92 steel substrate.

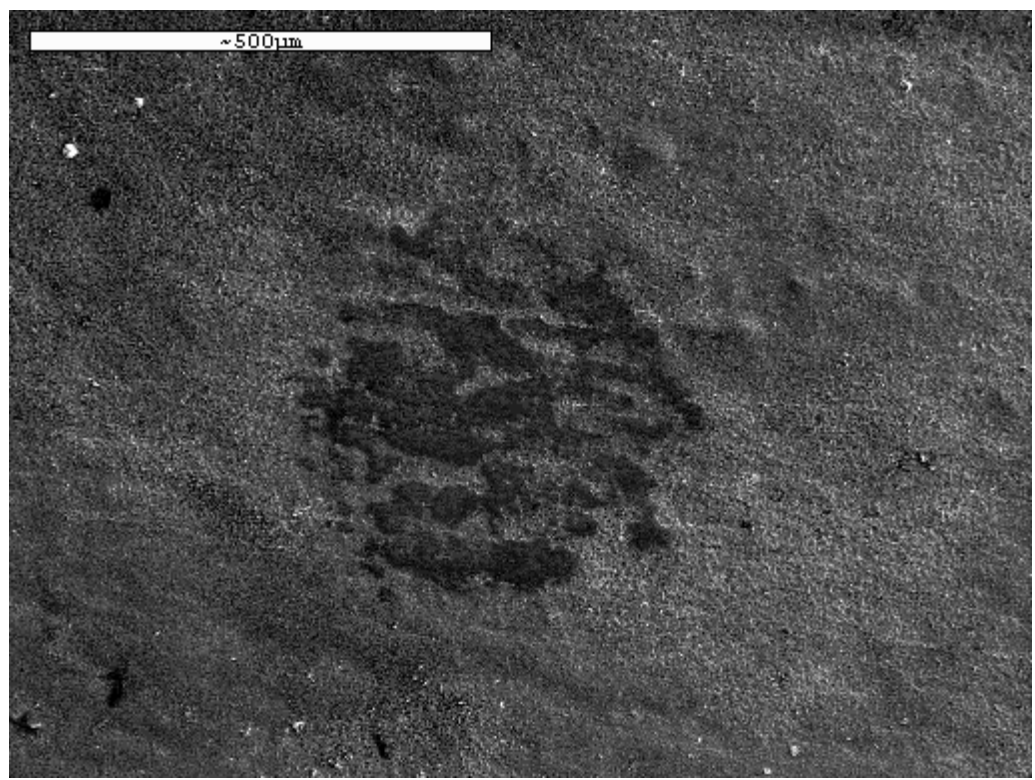

Figure 8: $\quad$ SEM photo of the Al, Fe coating deposited on P92 steel substrate after 1.000.000 impacts with an impact force of $100 \mathrm{~N}$, failure initiation. 


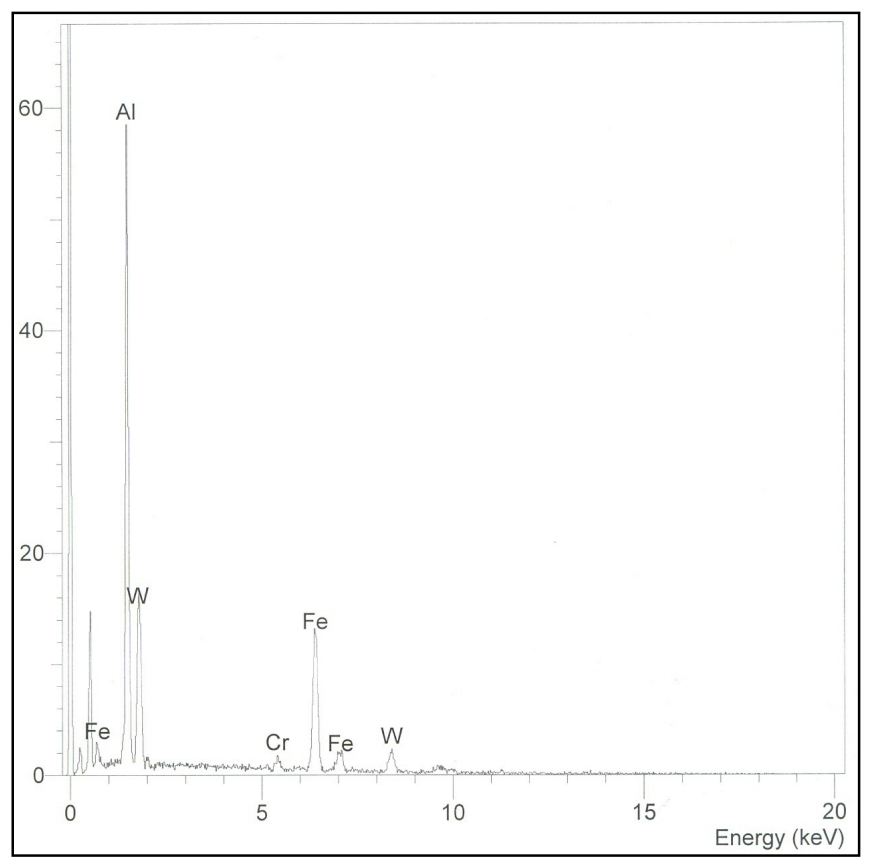

Figure 9: EDX diagram of the Al, Fe coating deposited on P92 steel substrate after 1.000.000 impacts with an impact force of $100 \mathrm{~N}$. Traces of W and $\mathrm{Cr}$ indicates failure initiation.

\section{Acknowledgements}

We express our gratitude to the E.U. for financing this work through the project SUPERCOAT, Contract No: ENK5-CT-2002-00608 and to Technical University of Serres also.

\section{References}

[1] Voevodin A.A., Bantle R., Matthews A., Dynamic impact wear of TiCXNY and Ti-DLC composite coatings, Wear, 185 (1995), pp. 151157.

[2] Bantle R., Matthews A., Investigation into the impact wear behaviour of ceramic coatings, Surface and Coatings Technology, 74 -75 (1995), pp. 857-868.

[3] Heinke W., Leyland A., Matthews A., Berg G., Friedrich C., Broszeit E., Evaluation of PVD nitride coatings, using impact, scratch and Rockwell-C adhesion tests, Thin Solid Films, 270 (1995), pp. 431-438.

[4] Ziegele H., Rebholz C., Voevodin A.A., Leyland A., Rohde S. L., Matthews A., Studies of the tribological and mechanical properties of laminated $\mathrm{CrC}-\mathrm{SiC}$ coatings produced by r.f. and d.c. sputtering, Tribology International, Vol. 30, No. 12 (1997), pp.845-856. 